九州大学学術情報リポジトリ

Kyushu University Institutional Repository

Reproductive Isolation between Japanese and Myanmar Populations of Cotesia vestalis (= plutellae) (Hymenoptera: Braconidae), a Larval Parasitoid of Plutella xylostella (Lepidoptera: Plutellidae)

Htwe, Ah Nge

Institute of Biological Control, Graduate School of Bioresource and Environmental Sciences, Kyushu University

Takagi, Masami

Laboratory of Insect Natural Enemies, Division of Biological Control, Department of Applied Genetics and Pest Management, Faculty of Agriculture, Kyushu University

Takasu, Ke i j i

Faculty of Agriculture, Kyushu University

https://doi.org/10.5109/14052

出版情報：九州大学大学院農学研究院紀要. 54 (1)，pp.153-157，2009-02-27. Faculty of Agriculture, Kyushu University

バージョン :

権利関係 : 


\title{
Reproductive Isolation between Japanese and Myanmar Populations of Cotesia vestalis (= plutellae) (Hymenoptera: Braconidae), a Larval Parasitoid of Plutella xylostella (Lepidoptera: Plutellidae)
}

\author{
Ah Nge HTWE ${ }^{1}$, Masami TAKAGI ${ }^{2}$ and Keiji TAKASU ${ }^{2}$ \\ Laboratory of Insect Natural Enemies, Division of Biological Control, Department of Applied \\ Genetics and Pest Management, Faculty of Agriculture, Kyushu University, \\ Fukuoka 812-8581, Japan \\ (Received November 14, 2008 and accepted December 5, 2008)
}

\begin{abstract}
Cotesia vestalis (Hymenoptera: Braconidae) has been widely used in many parts of the world as a major biocontrol agent of the diamondback moth, Plutella xylostella. The rate of parasitism and the rate of control exerted on the pests are highly variable geographically as well as not all introduction and augmentations were successful. Populations from different geographic regions often display various levels of reproductive incompatibility because they have been geographically isolated, experienced different selection pressures and/or adapted to various local environments. Experiment were conducted to compare reproductive compatibility between two geographic populations of C. vestalis one from Fukuoka, Japan (Temperate population) and the other from Mandalay, Myanmar (Tropical population). Results indicated that there was partial incompatibility between the two populations.
\end{abstract}

\section{INTRODUCTION}

Populations from different geographic regions often display various levels of reproductive incompatibility and exhibit different biological traits and genetic variability because they have been geographically isolated, experienced different selection pressures and/or adapted to various local environments (Diehl and Bush, 1984; Hopper et al., 1993). Cotesia vestalis Haliday (= C. plutellae) (Hymenoptera: Braconidae) is a solitary endo-larval parasitoid of diamondback moth (DBM), Plutella xylostella (Talekar and Yang, 1993; Noda et al., 1996; Kawaguchi and Tanaka, 1999), a serious pest of cruciferous crops worldwide (Abro et al., 1992; Talekar and Shelton, 1993; Reddy et al., 2004; Shelton, 2004). This parasitoid is distributed worldwide (Lim, 1986; Waterhouse and Norris, 1987; Talekar and Shelton, 1993; Talekar and Yang, 1993) and has been used in biological control programs of DBM in several countries because of its resistance against insecticides (Waterhouse and Norris, 1987; Fitton and Walker, 1992). This parasitoid has been widely used as a biological control agent for DBM and it is one of the most important agents of diamondback moth control in Southeast Asia (Lim, 1992; Talekar, 2004).

Although C. vestalis distributed world wide, it is currently reintroduced in already colonized areas, because a number of attempts to introduce and reintroduce failed (Waterhouse and Norris, 1987; Waterhouse, 1992). Major factors of these failures may have been a lack of knowledge of the biology and ecology of the introduced popula-

\footnotetext{
Institute of Biological Control, Graduate School of Bioresource and Environmental Sciences, Kyushu University, Fukuoka 812-8581, Japan

2 Faculty of Agriculture, Kyushu University, Fukuoka 812-8581, Japan

* Corresponding author (E-mail: mtakagi@grt.kyushu-u.ac.jp)
}

tions, and reproductive incompatibilities between local and introduced (or among introduced) populations (Rincon et al., 2006). Wang and Keller (2002) categorized $C$. vestalis a generalist parasitoid and there was highly variable in rates of parasitism of the population of C. vestalis and the level of control exerted on the pest geographically (Goudegnon et al., 2004; Guilloux et al., 2003; Kfir, 1997; Waladde et al., 1999).

The possible reproductive isolation between populations of natural enemies has not received much attention from researchers in the context of classical biological control (Rincon et al., 2006). The effect of releasing a nonnative species might not have much of an influence on biological control if no interspecific mating occurs between the released and native population (Stouthamer et al., 2000). The introduction of reproductively incompatible biological control agents may have severe negative effect on the outcome of biological control operations, even leading to the extinction of the local populations of natural enemies (Rincon et al., 2006). Partial reproductive isolation can potentially reduce fertility due to post-mating incompatibilities and/or reduce progeny viability by hybrid depression (Turelli et al., 2001).

Reproductive incompatibility in arrhenotokous Hymenoptera is measured using the production of females in inter-population crosses (Pinto et al., 1991, Stouthamer et al., 1996) because only females result from fertilized eggs, whereas males originate from unfertilized eggs. The lack of females in between-species crosses can have several causes: (1) no insemination takes place in the between-species crosses, (2) insemination takes place but males develop from fertilized eggs, (3) fertilized eggs die and the all-male offspring produced from unfertilized eggs. These various outcomes can have profound effects on biological control when one species is released in the native area of another (Stouthamer et al., 2000).

Rinconon et al. (2006) studied the reproductive com- 
patibility between 5 populations of $C$. vestalis from (South Africa, Benin, Martinique, Reunion and Taiwan).They found that the five populations represent two geographically widespread reproductive groups that are partially incompatible with one another and concluded that $C$. vestalis is a single species composed of at least two partially incompatible population aggregates. Comparative studies between its geographic population would help to reveal intraspecific variation which, if exist, should be considered in the introduction of this parasitoid for the biological control. Here we examined the reproductive compatibility of two geographic populations of C. vestalis, one from Japan and the other from Myanmar.

\section{MATERIALS AND METHODS}

\section{Cotesia vestalis culture}

Two geographic populations of $C$. vestalis were used in this study: one originating from Hisayama, Fukuoka Prefecture, Japan in April 2007 and the other from TadarOo City, Mandalay Township, Myanmar in May 2006. Both parasitoid populations were collected from DBM larvae on farmers' cabbage field. Myanmar population was started with about 200-300 cocoons and Japanese population was started with about 100 cocoons and 100 parasitized $P$. xylostella larvae. The identification of $C$. vestalis both Japanese and Myanmar populations were taxonomically confirmed by Dr. Kaoru Maeto (Laboratory of Insect Science, Faculty of Agriculture, Kobe University, Japan). Parasitoids were maintained on P. xylostella larvae fed on artificial diet in laboratory. DBM larvae were obtained from a stock culture maintained at Kyushu University, Japan. The laboratory colony of DBM was originated from field collected (about 200-300) larvae and pupae from farmers' cabbage field in Hisayama, Fukuoka Prefecture, Japan at April 2004 and maintained on artificial diet (Htwe et al., in preparation). Two populations of the parasitoid were reared in separate room at $25^{\circ} \mathrm{C}$ and 16L: 8D.

\section{Crossing experiments}

To obtain virgin females and males from the two populations of $C$. vestalis, DBM larvae were exposed to adult wasps for $24 \mathrm{hr}$ for oviposition. The parasitized larvae were reared at $25^{\circ} \mathrm{C}$ for parasitoid development and the parasitoid cocoons were singly placed in $1.5 \mathrm{ml}$ centrifuge tube after pupation until adult emergence. We set up a total of 25 crosses for each combination. The crossing experiment consisted of homogamic control crosses (intra-population crosses) and heterogamic crosses (interpopulation crosses). The two intra-population crosses and two inter-population crosses were conducted concurrently at $25^{\circ} \mathrm{C}$ with a 16L: $8 \mathrm{D}$ photoperiod. Newly emerged male and female wasps less than $6 \mathrm{hr}$ old were used in crossing experiments. One virgin female and two virgin males from the corresponding strains were enclosed in a glass tube $(7.5 \mathrm{~cm}$ height $\times 1.5 \mathrm{~cm}$ diameter $)$ for $24 \mathrm{hr}$ providing with honey streak. The parasitoid couples were then each provided with 50 early third instar DBM larvae for $24 \mathrm{hr}$. Parasitized larvae were maintained on artificial diet until the production of pupae and adults of $C$. vestalis or until caterpillar pupation. Parasitized larvae were monitored daily and newly developed cocoons were collected and held singly in $1.5 \mathrm{ml}$ centrifuge tube until adult emergence. The numbers of offspring and their sex were determined. Due to their haplodiploid sex determination system, a cross was considered incompatible in the absence of female in the progeny. Reproductive compatibility was calculated as the proportion of couples that produced at least one female, in each direction. The number and sex ratio of progeny from different combinations were compared to estimate the levels of reproductive compatibility between the two populations.

Statistical analysis: Data for total numbers of progeny were analyzed using one way analysis of variance (ANOVA) and means were separated by Tukey's HSD test. Sex ratios were transformed by arcsine square root and analyze by Bonferroni test. Data for compatibility rate between and within population crosses were analyzed by Fisher's Exact Probability test by using R 2. 8. 1 (R Development Core Team 2008). Statistical analyses were done using the statistical software package, Stat View (SAS Institute, 1998).

\section{RESULTS}

Reproductive compatibility between Japanese and Myanmar C. vestalis population was estimated from reciprocal crossing experiments. Table 1 showed the compatibility rate, number of female progeny, number of total progeny and sex ratio of progeny obtained for all crosses. Compatibility rates were higher in homogamic crosses (intra-population crosses) than in heterogamic crosses (inter-population crosses). The highest compatibility rate was observed in Myanmar $+\times$ Myanmar o (homogamic cross) (92\%) which was not significantly low $(P=0.84)$ and the compatibility rate in Japanese $+9 \times$ Japanese $\sigma^{\top}$ (homogamic cross) was 80\%. There was only 12\% compatibility in both (Myanmar $+\times$ Japanese $\sigma^{7}$ ) and (Japanese $+\times$ Myanmar $\sigma^{7}$ ) heterogamic crosses. Sex ratio was significantly male biased for females mated with males of the different population (inter-populations) $(p<0.01)$ than for female mated with males of the same population (intra-population) $(p<0.01)$ (Table 1). For compatible couples, the sex ratio of the progeny was nearly (1:1) for both intra- and inter-population crosses except in (Myanmar 우 $\times$ Japanese $\sigma^{\top}$ ) heterogamic cross.

Total number of progeny was higher in (Myanmar 우 $\times$ Myanmar $^{\top}$ ) homogamic cross than (Japanese 우 $\times$ Japanese $\sigma^{7}$ ) homogamic cross (ANOVA, $F_{3,96}=2.77, P=$ 0.046), however, no significant differences were observed for number of total progeny between intra- and interpopulations crosses (Table 1). Although total number of progeny was not significantly difference between intraand inter-populations cross, both inter-population crosses resulted in very few female than intra-population crosses (ANOVA, $F_{3,96}=15.26, P<0.01$ ). 
Table 1. Reproductive compatibility between Japanese and Myanmar populations of C. vestalis

\begin{tabular}{|c|c|c|c|c|c|c|}
\hline Cross $^{a}$ & $\begin{array}{l}\text { Number of } \\
\text { crosses } \\
(\mathrm{n} 1, \mathrm{n} 2)^{\mathrm{b}}\end{array}$ & $\underset{\text { rate }^{c}}{\text { Compatibility }}$ & $\begin{array}{c}\text { Number of } \\
\text { Total } \\
\text { progeny }^{\mathrm{d}}\end{array}$ & $\begin{array}{c}\text { Number of } \\
\text { female } \\
\text { Progeny }\end{array}$ & $\begin{array}{l}\text { sex ratio } \\
\left(\begin{array}{l}\left(\text { 우 }+0^{7}\right) \\
(\text { overall })^{\mathrm{f}}\end{array}\right.\end{array}$ & 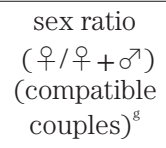 \\
\hline MM우 $\times \mathrm{MM}^{7}$ & 25,23 & $0.92 \mathrm{a}$ & $32.32 \pm 2.39 \mathrm{a}$ & $14.56 \pm 1.93 \mathrm{a}$ & $0.45 \pm 0.04 \mathrm{a}$ & $0.48 \pm 0.04 b$ \\
\hline $\mathrm{MM}$ 우 $\times \mathrm{JP}^{7}$ & 25,3 & $0.12 \mathrm{~b}$ & $25.52 \pm 2.66 \mathrm{ab}$ & $3.04 \pm 1.87 \mathrm{bc}$ & $0.11 \pm 0.06 \mathrm{~b}$ & $0.81 \pm 0.01 \mathrm{a}$ \\
\hline $\mathrm{JP}$ 우 $\times \mathrm{JP}^{7}$ & 25,20 & $0.80 \mathrm{a}$ & $22.88 \pm 2.39 \mathrm{~b}$ & $7.12 \pm 1.30 \mathrm{~b}$ & $0.35 \pm 0.05 \mathrm{a}$ & $0.43 \pm 0.05 b$ \\
\hline $\mathrm{JP}$ 우 $\times \mathrm{MM}^{\mathrm{T}}$ & 25,3 & $0.12 \mathrm{~b}$ & $26.32 \pm 2.12 \mathrm{ab}$ & $1.00 \pm 0.71 \mathrm{c}$ & $0.04 \pm 0.01 \mathrm{~b}$ & $0.31 \pm 0.14 b$ \\
\hline
\end{tabular}

${ }^{a}$ MM, Myanmar population and JP, Japanese population.

${ }^{\mathrm{b}} \mathrm{n} 1=$ total numbers of crosses, $\mathrm{n} 2=$ numbers of cross that produce both female and male progeny.

${ }^{c}$ Means followed by the same letter do not differ significantly from each other by Fisher's Exact Probability test, $P<0.01$ )

${ }^{\mathrm{d}, \mathrm{e}} \mathrm{M}$ Means followed by the same letter do not differ significantly from each other by Tukey's HSD test after one way ANOVA, $P<0.05$.

${ }^{\mathrm{f}, \mathrm{g}}$ Means followed by the same letters within the same column do not differ significantly from each other by Bonferroni test $P<0.01$

\section{DISCUSSION}

The results of crossing experiment clearly showed that reproductive isolation exists between Japanese and Myanmar populations of $C$. vestalis. The results of the present work rely on the estimation of reproductive incompatibility based on sex ratio data of a haplodiploid species. Heterogamic crosses between Japanese and Myanmar population produced only a few female offspring. In haplodiploid insect species, incompatibility in reproduction between strains results in skewed, highly male-biased sex ratios and reduced progeny production (Diel and Bush, 1984; Pinto et al., 1991). Reciprocals crosses between Japanese and Myanmar populations resulted highly mail-biased sex ratio in our experiment.

Many taxa used in biological control may be composed of several population units reproductively isolated to various degrees. In our experiment although, compatibility rate was higher in homogamic crosses, but both heterogamic crosses (Japanese 우 $\times$ Myanmar $\sigma^{\top}$ and Myanmar + × Japanese $\sigma^{7}$ ) had very low compatibility rate. The introduction of a population reproductively incompatible with the local population may cause a long term reduction of the levels of control of the pest and result in a depression of intended biological control (Mochiah et al., 2002; Stouthamer et al., 2000). It is therefore important for any biological control attempt to determine the reproductive compatibility between local and introduced populations or between different source populations of the natural enemy selected. This is also important for the establishment of laboratory cultures because mixing of different populations could result in a severe reduction of population growth in the laboratory (Rincon et al., 2006).

Populations of parasitoids collected from different host species or geographical regions can differ in host specificity. Host specificity surveys showed that $C$. vestalis can occasionally parasitise other species in the field, and laboratory studies in quarantine in New Zealand showed it has the ability to develop in several families of Lepidoptera (Cameron et al., 1998). In our study, the population of $P$. xylostella from Japan was used as hosts for both populations of $C$. vestalis. As Japanese popula- tion was collected from Japan, it would have had longer association with the host population than Myanmar Population. However, in crossing experiment C. vestalis from Myanmar population performed better than Japanese population in terms of total progeny as well as sex ratio. Therefore, it seems reasonable to assume that Myanmar population had better host suitability to $P$. $x y$ lostella than Japanese population. Furthermore, the larger wasp size in Myanmar population than Japanese population (Htwe et al., unpublished) could be resulted in higher progeny production in Myanmar population because lifetime reproductive success is positively correlated with body size (Jervis et al., 2001, 2003).

Rincon et al. (2006) stated that although multiple introductions of the same species of different origins seemed to be the best recipe for the successful introducing of biological control agents with the broadest genetic base possible is recommended but it can actually be a roadmap to failure. By the result of our experiment we agree with Rincon et al. (2006) statement that several introductions failures in the case of $C$. vestalis (Cordero and Cave, 1992; Waterhouse and Norris, 1987; Waterhouse, 1992) might be attributed to reproductive incompatibility of the strains selected for introduction or of the introduced strain with an already present one.

In some cases, cross mating incompatibility has been used to support cases where minor morphological, life history or allozymic differences have not been convincing enough to argue for species recognition (Pinto et al., 1991). In others, it comprises the primary or even the sole source of evidence for the species (Nagarkatti, 1975), which have been justified by the role played by reproductive isolation in speciation (Pinto et al., 1991) and the need to formally recognize distinct populations for biological control purposes. Lower reproductive compatibility between Japanese and Myanmar population in this study and smaller body size of Japanese population than Myanmar population (Htwe et al., unpublished) showed us further investigation were needed to speciation process. The infection statuses of Wolbachia (a causative agent of various reproductive changes in arthropods, induces cytoplasmic incompatibility), as well as the possible ecological differentiation between the Japanese and 
Myanmar populations of $C$. vestalis were needed to better understand the underlying mechanisms of reproductive isolation between the two populations.

The occurrence of reproductive isolation between the two geographically isolated populations of $C$. vestalis may have important consequences for the use of these species in inundative biological control. In Japanese and Myanmar strains of $C$. vestalis releasing both strains together could lead to a lower level of biological control than releasing either of the strains alone. It is importance to understand reproductive isolation between strains of biological control agents before their introduction (Stouthamer et al., 2000; Mochiah et al., 2002).

\section{ACKNOWLEDGMENT}

We thank Dr. Kaoru Maeto (Laboratory of Insect Science, Faculty of Agriculture, Kobe University, Japan) for taxonomic confirmation of $C$. vestalis both Japanese and Myanmar populations.

\section{REFERENCES}

Abro, G. H., R. A. Soomro and T. S. Syed 1992 Biology and behavior of diamondback moth, Plutella xylostella (L.) Pakistan J. Zoo., 24: 7-10

Biever, K. D., D. L. Hostetter and J. R Kernn 1994 Evolution and implementation of a biological control-IPM system for crucifers: 24 year case history. Am. Entomol., 40: 103-108

Cameron, P. J., P. J. Walker and M. A. Keller 1998 Comparative measures of the host specificity of Cotesia rubecula and $C$. plutellae (Hymenoptera: Braconidae). Proceedings of the Sixth Australasian Applied Entomological Research Conference, Brisbane, Australia, 29 September - 2 October 1998: 512-516

Cordero, J. and R. D. Cave 1992 Natural enemies of Plutella $x y$ lostella (Lepidoptera: Plutellidae) on crucifers in Honduras. Entomophaga., 37: 397-407

Diehl, S. R. and G. L. Bush 1984 An evolutionary and applied perspective of insect biotypes. Annu. Rev. Entomol., 29: $471-483$

Fitton, M. and A. Walker 1992 Hymenopterous parasitoids associated with diamondback moth: the taxonomic dilemma. In Management of Diamondback Moth and Other Crucifer Pests: Proceedings of the second international workshop. (N. S. Talekar ed.) pp. 225-232. Asian Vegetable Research and Development Center, Shanhua, Taiwan

Goudegnon, A. E., A. A. Kirk, L. Arvanitakis and D. Bordat 2004 Status of the Diamondback moth and Cotesia plutellae its main parasitoid in the Cotonou and Porto-Novo periurban areas of Benin. In: Kirk, A. A., Bordat, D. (Eds.), Improving biocontrol of Plutella xylostella: Proceedings of the International Symposium, Montpellier, France, 21-24 October, 2002, pp. $172-178$

Guilloux, T., R. Monnerat, M. Castelo-Branco, A. Kirk and D. Bordat 2003 Population dynamics of Plutella xylostella (Lep., Yponomeutidae) and its parasitoids in the region of Brasilia. $J$. Appl. Entomol. 127: 288-292

Hopper, K. R., R. T. Roush and W. Powell 1993 Management of genetics of biological control introductions, Annu. Rev. Entomol., 38: 27-51

Jervis, M. A., G. E. Heimpel and P. N. Ferns 2001 Lifehistory strategies in parasitoid wasps: a comparative analysis of 'ovigeny', $J$. Anim. Ecol., 70: 442-458

Jervis, M. A., P. N. Ferns and G. E. Heimpel 2003 Body size and the timing of egg production in parasitoid wasps: a comparative analysis. Funct. Ecol., 17: 375-383
Kawaguchi, M. and T. Tanaka 1999 Biological characteristics of a larval endoparasitoid, Cotesia plutellae (Hymenoptera: Braconidae): Host stage preference, subsequent sex ratio of progeny and mate location of males. Appl. Entomol. Zool., 34: 213-221

Kfir, R. 1997 Parasitoids of Plutella xylostella (Lepidoptera: Plutellidae) in South Africa: an annotated list. Entomophaga. 42: $517-523$

Lim, G. S. 1986 Biological control of diamondback moth Diamondback moth Management: Proceedings of the First International Workshop (eds. N. S. Talekar \& T. D. Griggs), March 11-15, Shanhua, Taiwan, China pp. 159-171

Lim, G. S. 1992 Integrated pest management of diamondback moth: practical realities. In: Talekar, N. S. (Ed.), Diamondback Moth and Other Crucifer Pests: Proceedings of the second International Workshop, Tainan, Taiwan, 10-14 December, 1990

Mochiah, M. B., A. J. Ngi-Song, W. A. Overholt and R. Stouthamer 2002 Wolbachia infection in Cotesia sesamiae (Hymenoptera: Braconidae) causes cytoplasmic incompatibility: implications for biological control. Biol. Control., 25: 74-80

Nagarkatti, S. 1975 Two new species of Trichogramma (Hymenoptera: Trichogrammatidae) from the USA. Entomophaga., 20: 245-248

Nakahara, L. M., J. J. McHugh, C. K. Otsuka, G. Y. Funasaki and P. V. Lai 1986 Integrated control of diamondback moth and other insect pests using an overhead sprinkler system, an insecticide, and biological control agents, on a watercress farm in Hawaii. In: Talekar, N. S. (Ed.), Diamondback Moth Management: Proceedings of the First International Workshop, Tainan, Taiwan. 11-15 March, 1985, pp. 403-413

Noda, T., S. Miyai and S. Yamada 1996 Larval and pupal parasitoids of diamondback moth, Plutella xylostella (L) in cabbage fields in Morioka, Japan. Japanese J. Appl. Entomol. Zool., 40 164-167

Pinto, J. D., R. Stouthamer, G. R. Platner, and E. R. Oatman 1991 Variation in reproductive compatibility in Trichogramma and its taxonomic significance (Hymenoptera: Trichogrammatidae). Ann. Entomol. Soc. Am., 84: 37-46

R Development Core Team 2008 R : A language and environment for statistical computing. R Foundation for Statistical Computing, Vienna, Austria. ISBN 3-900051-07-0, URL http:// www. R-project. org.

Reddy, G. V. P., E. Tabone, and M. T. Smith 2004 Mediation of host selection and oviposition behavior in the diamondback moth Plutella xylostella and its predator Chrysoperla carnea by chemical cues from cole crops. Biol. Cont., 29: 270-277

Rincon, C., D. Bordat, B. Lohr and S. Dupas 2006 Reproductive isolation and differentiation between five populations of Cotesia plutellae (Hymenoptera: Braconidae), parasitoid of Plutella xylostella (Lepidoptera: Plutellidae). Biol. Cont., 36: 171-182

SAS Institute. 1998 Stat View 5.0.J.SAS Institute, Cary, NC

Shelton, A. M. 2004 Management of the diamondback moth: déjà vu all over again? In: Endersby, N. M., Ridland, P. M. (Eds.), The Management of Diamondback Moth and Other Crucifer Pests Proceedings of the Fourth International Workshop, 26-29 November 2001. Department of Natural Resources and Environment, Melbourne, Australia, pp. 3-8

Stouthamer, R., P. Jochemsen, G. R. Platner and J. D. Pinto 2000 Crossing incompatibility between Trichogramma minutum and T. platneri (Hymenoptera: Trichogrammatidae): implications for applications in biological control. Environ. Entomol., 29: 832-837

Stouthamer, R., R. F. Luck, J. D. Pinto, G. R. Platner, and B. Stephens 1996 Non-reciprocal cross-incompatibility in Trichogramma deion. Entomol Exp. Appl., 80: 481-489

Talekar, N. S. 2004 Biological control of diamondback in Asia, In: A. A. Kirk, , D. Bordat (Eds.), Improving biocontrol of Plutella xylostella: Proceedings of the International Symposium, Montpellier, France, 21-24 October, 2002, pp. 103-113

Talekar, N. S. and A. M. Shelton 1993 Biology, ecology and man- 
agement of the diamondback moth. Annv. Rev. Entomol., 38: 275-301

Talekar, N. S. and J. C. Yang 1993 Influence of crucifer cropping system on the parasitism of Plutella xylostella (Lep., Yponomeutidae) by Cotesia plutellae (Hym., Braconidae) and Diadegma semiclausum (Hym., Ichneumonidae). Entomophaga., 38: 541-550

Turelli, M., N. H. Barton and J. A. Coyne 2001 Theory and speciation, Trends Ecol. Evol., 16: 330-343

Waladde, S. M., M. H. Villet, M. F. Leutle 1999 Parasitism on Plutella xylostella (Lepidoptera: Yponomeutidae): observations in the Eastern Cape Province, South Africa. In: van Rensburg J. B. J.; van den Berg J. (Eds), Proceedings of the twelfth Entomological Congress. Potchefstroom, 12-15 July, 1999, pp. 135

Wang, X. G and M. A. Keller 2002 A comparison of the hostsearching efficiency of two larval parasitoids of Plutella xylostella, Ecol. Entomol., 27: 105-114

Waterhouse, D. F. 1992 Biological control of diamondback moth in the Pacific, In: Talekar, N. S. (Ed.), Diamondback Moth and Other Crucifer Pests: Proceedings of the Second International Workshop, Tainan, Taiwan, 10-14 December, 1990

Waterhouse, D. F. and K. R. Norris 1987 Plutella xylostella (Linnaeus), In: The Australian Center for International Agricultural Research (Ed.), Biological Control: Pacific Prospects. Inkata Press, Melbourne, pp. 177-191 